\title{
The Canadian Cohort Obstructive Lung Disease (CanCOLD): New Insights for Primary Care Application
}

Jean Bourbeau, MD, FRCPC 1,2 , Wan Tan, MD ${ }^{3}$, Helene Perrault, $\mathrm{PhD}^{1,4}$. On behalf of Canadian Respiratory Research Network and the CanCOLD Collaborative Research group

${ }^{1}$ Respiratory Epidemiology and Clinical Research Unit, Department of Medicine, McGill University, Montreal, Quebec, Canada; ${ }^{2}$ Research Institute of McGill University Health Centre, Montreal, Quebec, Canada; ${ }^{3}$ Department of Medicine, University of British Columbia, Vancouver, British Columbia, Canada; ${ }^{4}$ Faculty of Health Sciences, University of Ottawa, Ottawa, ON, CANADA

\section{ABSTRACT}

The Canadian Cohort Obstructive Lung disease (CanCOLD) study was launched ten years ago, to provide insight on the natural course of chronic obstructive pulmonary disease (COPD). This project, unique on the world scene for its longitudinal evaluation, is based on random sampling of the population as opposed to more traditional cohorts built on convenience samples of clinical patients.

This comprehensive review article has elected to extract and collate from the set of CanCOLD substudies those findings and emerging discoveries that can serve to influence clinical practice, in particular, for primary care. We are presenting findings grouped around three aspects: prevalence of COPD, diagnostic performance, and impacts of COPD. Furthermore, the main results of these studies are supplemented by key messages.

From CanCOLD publications we have already learned much, with sufficient substantive data to warrant translation into our clinical practice, guidelines and health policies for the benefit of our COPD patients. (BRN Rev. 2019;5(4):249-62)

Corresponding author: Jean Bourbeau, jean.bourbeau@mcgill.ca

Key words: Chronic obstructive pulmonary disease. Exacerbations. Misdiagnosis. Smokers with normal spirometry. Underdiagnosis.

\section{Correspondence to:}

Jean Bourbeau MD, MSc, FRCPC

Respiratory Epidemiology and Clinical Research Unit

Research Institute of the McGill University Health Centre

5252 De Maisonneuve, room 3D.62

Received in original form: 28-06-2019

Montreal, QC, H4A 3S5, Canada

Accepted in final form: 07-08-2019

E-mail: jean.bourbeau@mcgill.ca

DOI: 10.23866/BRNRev:2019-0007 


\section{INTRODUCTION}

Stemming from the fact that there existed very few prospective and well-characterised population-based cohorts in chronic obstructive lung disease (COPD) to inform the medical community and provide substantive data for use in clinical practice with particular attention to the primary care setting, the Canadian Cohort Obstructive Lung Disease (CanCOLD) population-based study was launched almost ten years ago, to provide insight on the natural course of COPD and other related comorbid conditions.

The CanCOLD study takes its origin from the Burden of Obstructive Lung Disease (BOLD) ${ }^{1}$, a cross-sectional, general population-based survey in 11,985 subjects from 17 cities/countries, describing the impact of COPD. No country other than Canada has taken the extra steps to further study the Canadian Obstructive Lung disease (COLD) cross-sectional/prevalence of COPD with a longitudinal design with prospective follow-ups, detailed assessments using questionnaires, as well as detailed measurements of lung function, cardiorespiratory exercise testing, computerised tomography (CT) scan imaging and biological sampling. Build on the original Vancouver site, COLD expanded initially by adding four additional cities including Montreal, and then again in 2009 to include a total of nine collaborating sites across six provinces in Canada for the implementation of the CanCOLD longitudinal prospective evaluation.

This project, unique on the world scene, is based on random sampling of the population as opposed to more traditional cohorts built on convenience samples of patients seen in a clinical setting. This distinctive feature makes CanCOLD the first population-derived COPD cohort for characterising the progression of the disease from normality, or at-risk because of smoking exposure, on to spirometrically-defined COPD, including mild and/or early disease through moderate disease. Another important feature lies in the geographic spread of its population sample including representation from a diversity of cities set in diverse regions, from coast to coast across Canada.

The CanCOLD initiative has been and continues to serve as a prime driver for training future generations of clinicians, researchers and highly qualified research staff. It has attracted the interest and support from many partners from governments, the industry or other national and international academic institutions to develop novel strategies for improving the diagnosis and care of COPD patients in Canada and worldwide. The multifaceted perspectives in the CanCOLD data set have to date inspired over 60 sub-studies conducted on issues ranging from characterisation of the disease and its trajectory to the assessment of comorbidities, patient exacerbations, symptom and burden of disease on the use and costs of health care services and workforce productivity. In particular, it has allowed us to better assess the diagnostic performance of COPD in Canada, serving to elaborate recommendations for earlier diagnosis of the disease and better guidance for clinical practice from primary care to secondary care settings.

While a comprehensive review of all pertinent data was beyond the scope of this manuscript, we have elected to extract and collate from the set of CanCOLD sub-studies those 


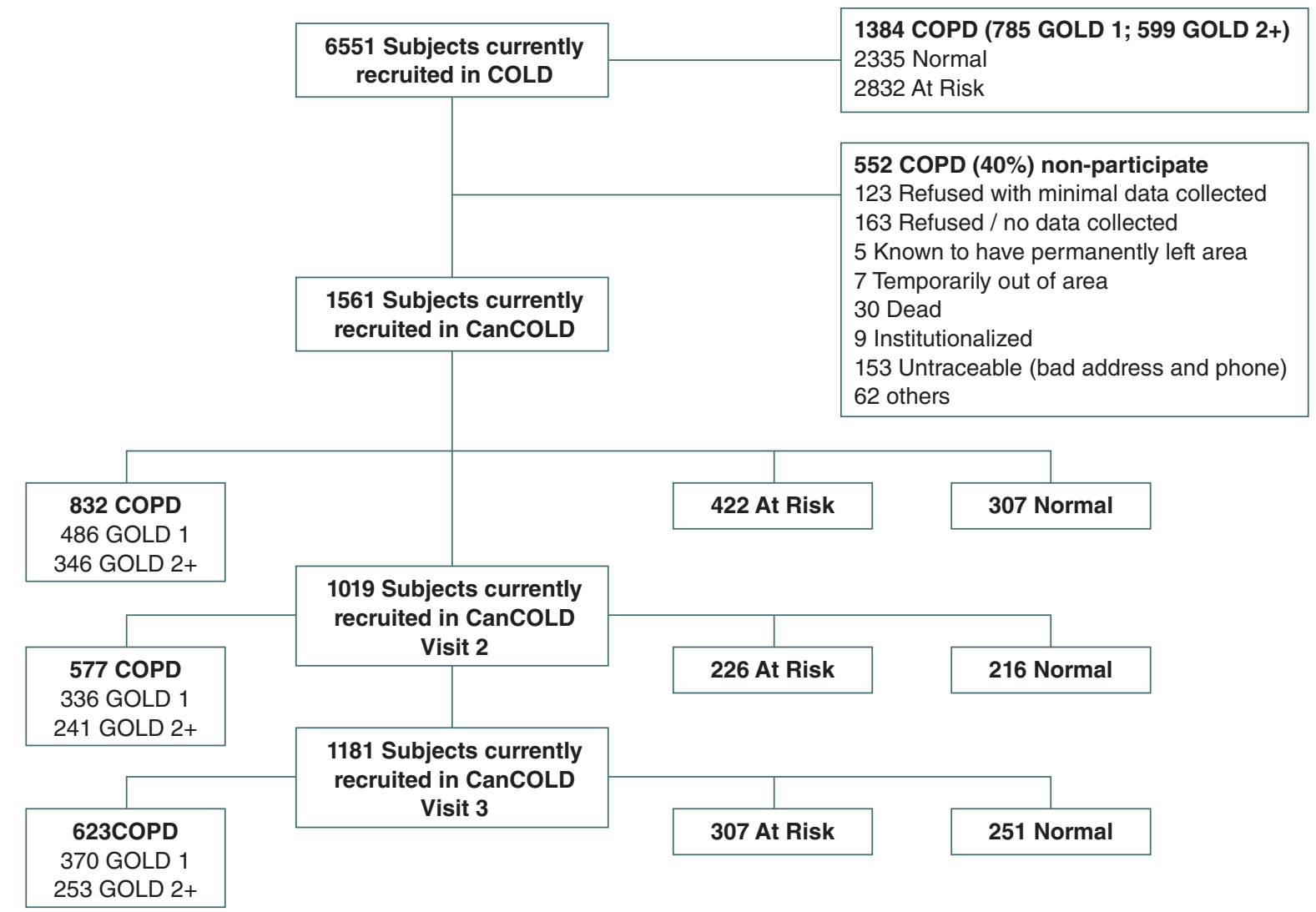

Figure 1. Flow diagram of the Canadian Obstructive Lung disease (COLD)/Canadian Cohort Obstructive Lung Disease (CanCOLD) study recruitment.

COPD: chronic obstructive pulmonary disease; GOLD: Global Initiative for Chronic Obstructive Lung Disease.

findings and emerging discoveries that can serve to influence and potentially change clinical practice with specific attention for its relevance to primary care practice for better disease management and patient wellbeing.

\section{METHODS}

In CanCOLD, individuals were recruited from the prevalence study COLD. The cross- sectional study COLD randomly sampled over 6500 individuals, non-institutionalised adults of $\geq 40$ years old, from nine Canadian cities
(Fig. 1). The COLD study participants were contacted to participate in the CanCOLD longitudinal study if they had airflow obstruction based on the Global Initiative for Chronic Obstructive Lung Disease (GOLD) criteria, i.e., the ratio forced expiratory volume in one second $\left(\mathrm{FEV}_{1}\right) /$ forced vital capacity (FVC) post bronchodilator $<0.70$. Then non-COPD peers (sex- and age- matched \pm 2 years) were recruited. More detailed information on the study protocol has already been published ${ }^{2}$.

Figure 1 shows detailed information about the recruitment and the follow-up visits. 


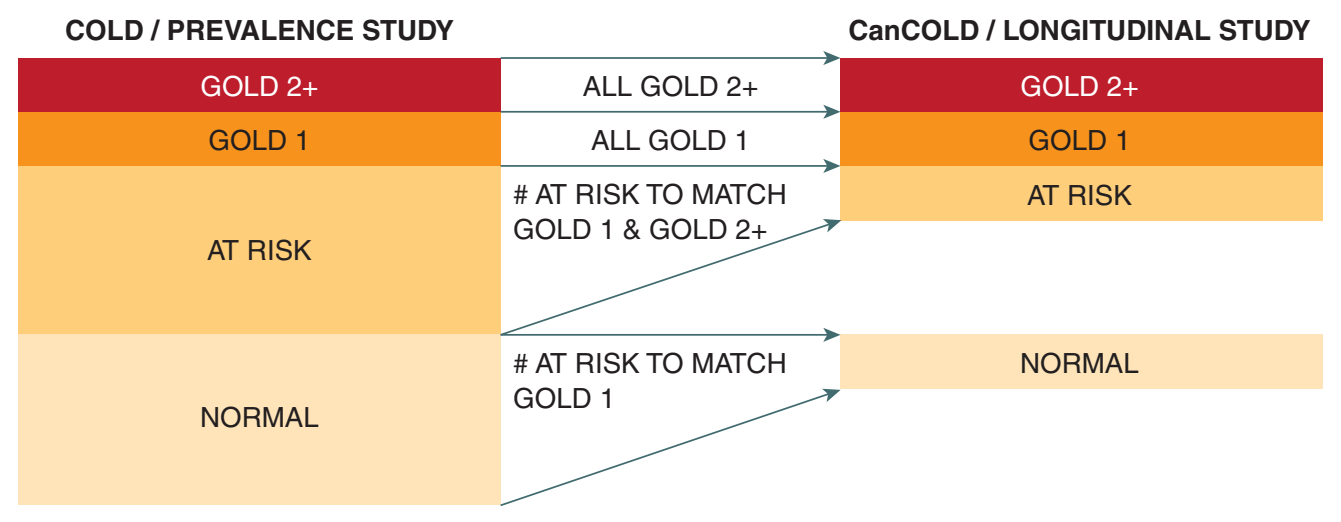

\begin{tabular}{lll} 
Visit 1 (complete) & Visit 2 (short) & Visit 2 (complete) \\
\hline 0 & 18 months & 18 months
\end{tabular}

Figure 2. Canadian Obstructive Lung disease (COLD)/Canadian Cohort Obstructive Lung Disease (CanCOLD) study design and procedures. A complete visit includes: core questionnaires on symptoms, medication, work and exposures, co-morbidities, health-related quality of life, sleep habits, nutrition and physical activities, spirometry pre- and post- bronchodilator, computed tomography scan of the chest, cardiopulmonary exercise test and blood samples; a short visit includes: same core questionnaires, six-minute walking test, complete pulmonary function tests and blood samples (adapted from Bourbeau J, et al. ${ }^{2}$ with permission from Tayylor \& Francis Ltd.).

GOLD: Global Initiative for Chronic Obstructive Lung Disease.

Among the 1384 COPD individuals in the COLD study, 832 (60\%) responded favourably and were enrolled in CanCOLD. A total of 1561 individuals were recruited, 486 GOLD 1, 301 GOLD 2, 45 GOLD 3+, and 422 "at risk" (smoker or ex-smoker with normal spirometry) and 307 healthy individuals, i.e., never smoker with normal spirometry.

Participants in CanCOLD have been followed prospectively and were assessed at baseline (visit 1 between 2009-2015), after 18 months but not for the whole initial cohort (visit 2 between 2011-2015) and three years for the whole initial cohort (visit 3 between 20152019). The attrition rate at visit 3 is of $\cong 20 \%$; 341 participants were lost in follow-up including 33 deaths. The most common reason for "dropping out" is withdrawal, reflecting subjects voluntarily declining to participate further in the study.

Figure 2 shows the CanCOLD visits with detailed data collection including sociodemographic, clinical, comorbidities, medication, health status, lifestyle and smoking and occupation risk factors and measurements from spirometry and full pulmonary function tests, cardiopulmonary exercise tests, computed tomography (CT) scan of the chest and venous blood samples for blood counts, plasma, serum, deoxyribonucleic acid (DNA) and ribonucleic acid (RNA) analyses. Throughout the follow-up, a COPD exacerbation questionnaire that included questions on changes in respiratory symptoms adjustment and/or new medication 

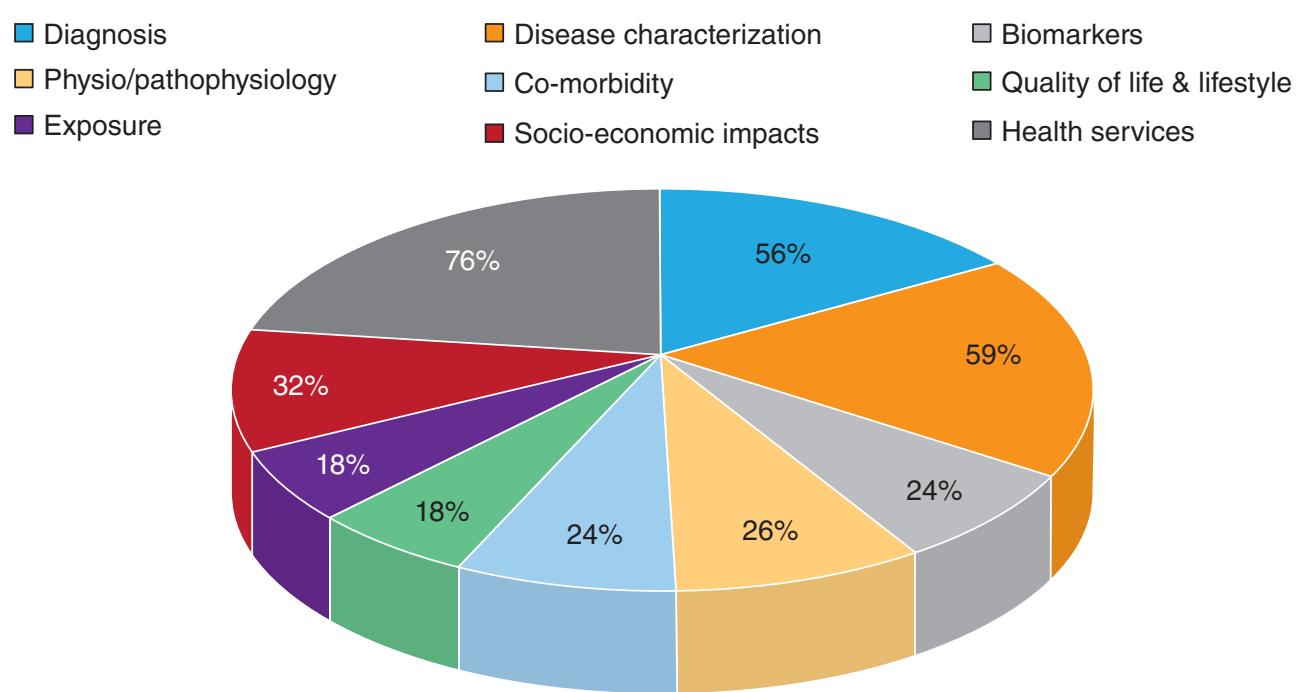

Figure 3. Canadian Obstructive Lung disease (COLD)/Canadian Cohort Obstructive Lung Disease (CanCOLD) research contribution domains.

and health service use (physician visits, emergency department visits and hospital admissions) was administered by telephone every three months. Finally, CanCOLD also gives the possibility to access provincial health administrative databases and confirm health service use.

\section{RESULTS}

Over the last decade, the comprehensive pan-Canadian multi-year cohort dataset has resulted in many COLD/CanCOLD-related peer-reviewed publications. To date, the publications have significantly advanced knowledge, contributing key findings serving as bases for emerging discoveries, approaches or technologies in a large number of domains (Fig. 3). In turn, the consolidation of these observations can translate into key messages for clinicians, industry partners and policymakers.
The largest contribution of COLD/CanCOLD-related publications may be seen to have advanced knowledge and understanding surrounding appropriate diagnostic approaches and disease characterisation with impact on recommendations for health care services and interventions. More specifically, the larger proportion of studies to date have served to appropriately quantify COPD prevalence and disease determinants ${ }^{3,4}$ spirometry testing and prediction equation $s^{5-9}$, diagnosis performance and early detection ${ }^{10-15}$, novel methods of assessing COPD ${ }^{16,17}$, and COPD clinical impact ${ }^{18-22}$. Consequently, we have elected for this manuscript to summarise key learnings from the CanCOLD study that are relevant to the primary care setting. These findings are grouped around three aspects: prevalence of COPD, diagnostic performance, and impacts of COPD (exacerbations, symptom burden and health-related quality of life). This is presented 
TABLE 1. Emerging discoveries and key learnings that could change clinical practice

\begin{tabular}{|c|c|}
\hline Discovery topic & Key learning for the physician \\
\hline \multicolumn{2}{|l|}{ Prevalence of COPD } \\
\hline $\begin{array}{l}\text { Underdiagnosis in national } \\
\text { surveys }\end{array}$ & $\begin{array}{l}\text { Important to reaffirm the need for spirometrically measured prevalence as the benchmark for accurate national data } \\
\text { on disease prevalence; COPD is a much larger health issue in developed countries than had been previously realised }\end{array}$ \\
\hline COPD in never-smokers & $\begin{array}{l}\text { COPD in never-smokers represents a substantial health burden in the population requiring special attention in clinical } \\
\text { practice; there are gender-specific differences in never smokers with COPD }{ }^{4} \text {. }\end{array}$ \\
\hline \multicolumn{2}{|l|}{ Diagnosis of COPD } \\
\hline $\begin{array}{l}\text { Diagnostic instability and } \\
\text { reversals in mild COPD }\end{array}$ & $\begin{array}{l}\text { More than a single post-bronchodilator spirometric assessment is necessary for diagnosing COPD, in particular for } \\
\text { patients with mild airflow obstruction at baseline }{ }^{10} \text {. }\end{array}$ \\
\hline $\begin{array}{l}\text { Debate around using the } \\
\text { fixed ratio versus LLN }\end{array}$ & $\begin{array}{l}\text { A diagnosis established by both a low } \mathrm{FEV}_{1} / \mathrm{FVC} \text { (according to fixed ratio and/or LLN) and a low } \mathrm{FEV}_{1} \text { is strongly } \\
\text { associated with poor clinical outcomes. }\end{array}$ \\
\hline COPD misdiagnosis & $\begin{array}{l}\text { COPD misdiagnosis be "un" or "over" diagnosis contributes significantly to health care burden and should be given } \\
\text { proper attention by the physician considering that proper treatment will have an impact on the patient and the } \\
\text { health care system }{ }^{13} \text {. }\end{array}$ \\
\hline \multicolumn{2}{|l|}{ Impact of COPD } \\
\hline $\begin{array}{l}\text { Undiagnosed COPD and } \\
\text { exacerbations }\end{array}$ & $\begin{array}{l}\text { Despite experiencing fewer exacerbations, health care uses to treat "exacerbation-like events" in undiagnosed } \\
\text { individuals with COPD is similar to that of diagnosed individuals. There is a missed opportunity by the physician to } \\
\text { recognize these patients, make a diagnosis of COPD and treat them properly to prevent further exacerbations }{ }^{20} \text {. }\end{array}$ \\
\hline \multicolumn{2}{|c|}{ Symptom burden and quality of life } \\
\hline CAT and mild COPD & $\begin{array}{l}\text { CAT, originally designed for use in clinically symptomatic COPD patients, can also be used in individuals with mild } \\
\text { airflow obstruction }{ }^{19} \text {. }\end{array}$ \\
\hline $\begin{array}{l}\text { Importance of assessing } \\
\text { symptom burden in mild } \\
\text { COPD }\end{array}$ & $\begin{array}{l}\text { In COPD individuals with mild airflow obstruction, those with a high symptom burden (CAT } \geq 10 \text { ), experience increased } \\
\text { work productivity loss (i.e. combined absenteeism and presenteeism) compared to COPD subjects with a low } \\
\text { symptom burden (CAT }<10 \text { ), and non-COPD subjects }{ }^{18} \text {. }\end{array}$ \\
\hline $\begin{array}{l}\text { Importance of psychologi- } \\
\text { cal distress in COPD }\end{array}$ & $\begin{array}{l}\text { Screening for psychological distress and developing interventions that target these health risk behaviours in this } \\
\text { population may be important for improving management and outcomes of COPD, event those with mild to moderate } \\
\text { disease }^{21} \text {. }\end{array}$ \\
\hline
\end{tabular}

CAT: COPD assessment test; COPD: chronic obstructive pulmonary disease; FEV ${ }_{1}$ : forced expiratory volume in one second; FVC: forced vital capacity; LLN: lower limit of normal.

in table 1 as a summary with overall key clinical messages.

\section{Epidemiology "distribution and determinants" of chronic obstructive pulmonary disease}

Despite the complexities inherent to the understanding of large health-related data sets, new approaches are emerging to enable more accurate estimates of COPD prevalence. Although cigarette smoking is the most studied risk factor for COPD, it is not the only risk factor. Recently, epidemiology studies have turned their attention to explore COPD in "never smokers".

Problem assessing the prevalence of COPD in national surveys. In the present population-based study CanCOLD ${ }^{3}$, overall, 16.7\% (95\% confidence interval [CI] 14.8-18.7) of study subjects met the criteria for GOLD severity 1 or higher, based on the fixed ratio post-bronchodilator $\mathrm{FEV}_{1} / \mathrm{FVC}$. Prevalence of COPD varied by severity across sites $(\mathrm{p}=0.0025)$. However, after 
age-sex adjustment, the variation disappeared $(\mathrm{p}>0.16)$. The spirometry-measured COPD prevalence was at least 2.0 times higher than the self-reported diagnosis in the same study and (three- to four-fold) higher than in national surveys.

Chronic obstructive pulmonary disease in never smokers. The occurrence of COPD in never-smokers has been recognised as a significant problem in developing countries and recently more attention has been given in developed countries. In CanCOLD, prevalence of COPD in never smokers was $6.4 \%$ and $15.2 \%$ in ever smokers; with "never smokers" accounting for $27 \%$ of all COPD subjects ${ }^{4}$. The common independent predictors were older age, self-reported asthma and lower education. In "never-smokers" a history of hospitalisation in childhood for respiratory illness was discriminative, while exposure to passive smoke and biomass fuel for heating were discriminative for women only. Furthermore, COPD in "never-smokers" and "ever-smokers" was characterised by increased respiratory symptoms, "respiratory exacerbation" events and increased residual volume/total lung capacity, but only smokers had reduced diffusing capacity for carbon monoxide divided by the alveolar volume (DLCO/VA) and emphysema on chest CT scans.

\section{Diagnosis of chronic obstructive pulmonary disease}

Spirometry should play the lead role in establishing a COPD diagnosis. The diagnosis is based on post-bronchodilator measurement of $\mathrm{FEV}_{1} / \mathrm{FVC}$, which is used to define expiratory airflow limitation. Two criteria have been used to diagnose COPD: 1) the fixed ratio, i.e., a post-bronchodilator $\mathrm{FEV}_{1} / \mathrm{FVC}<0.70$ or 2 ) the lower limit of normal (LLN) ratio, i.e., a post-bronchodilator $\mathrm{FEV}_{1} / \mathrm{FVC}$ ratio $<$ than the lower fifth percentile of the reference value matched to a healthy population.

Diagnostic instability and reversals of COPD diagnosis based on spirometric criteria. Diagnostic reversals of COPD (defined as a subject who met criteria for diagnosis of COPD at the study outset but whose spirometry had reversed to normal by the end of the study) occurred in the CanCOLD study in $27.2 \%$ (95\% CI, 21.2-33.8\%) of individuals from the beginning to the end of the 4-year study when the LLN criterion was used and in $21.5 \%$ (95\% CI, 17.2-26.3\%) when the fixed ratio was used $^{10}$. The prevalence of both diagnostic instability and reversal was higher for subjects who entered the study with GOLD 1 severity than for subjects with GOLD 2 severity $(19.7 \%$ versus $10.1 \%, p=0.001 ; 11.6 \%$ versus $4.8 \%$, $\mathrm{p}=0.001$, respectively).

Debate around using the fixed ratio versus LLN. The debate around the two main spirometric assessment criteria of the fixed ratio and the LLN for diagnosis of COPD has been ongoing for some time. The CanCOLD study provided us with a large population-based database to further explore the clinical relevance of these criteria in mild COPD 9 . Compared with peers who met neither criterion for airflow limitation, patients having airflow limitation based on fixed ratio only were significantly more likely to have physician-diagnosed wheeze (odds ratio $[\mathrm{OR}]=1.54$ ) and cardio-vascular disease $(\mathrm{OR}=1.52)$. In contrast, patients who met both the fixed ratio and LLN criteria for airflow limitation were at significantly increased 
risk for seven of the outcomes studied, with particularly strong associations seen for wheeze $(\mathrm{OR}=3.25)$, chronic bronchitis $(\mathrm{OR}=3.14)$, and chronic phlegm $(\mathrm{OR}=3.04)$. Additionally, these patients meeting both criteria also had elevated risk when compared with peers meeting the fixed ratio criterion alone. The addition of a low $\mathrm{FEV}_{1}(<80 \%$ predicted) to either or both criteria greatly strengthened the associations; patients having such airflow limitation had 2 to 5 times higher odds of most adverse outcomes relative to counterparts having no airflow limitation.

Misdiagnosis of COPD and the use of health care services. Misdiagnosis of COPD, i.e., "un" and "over" -diagnosis, is considered to be a common problem and the impact on the health care system remains incompletely documented. Using CanCOLD and the provincial health administrative databases, it was possible to quantify the health services burden of undiagnosed and overdiagnosed COPD in a real-world ${ }^{13}$. Fourteen percent $(13.7 \%)$ had undiagnosed COPD, 5.1\% were over diagnosed, and $3.7 \%$ had correctly diagnosed COPD. Subjects with overdiagnosed COPD had significantly higher rates of hospitalisations, emergency department visits, and ambulatory care visits, and subjects with moderate-to-severe undiagnosed COPD had higher rates of hospitalisations, than subjects in the non-COPD population.

\section{Impact of chronic obstructive pulmonary disease: exacerbations}

The natural course of COPD is often disturbed by episodes of acute symptom worsening, known as exacerbations. Little is known about such events in individuals with undiagnosed COPD in the general population and their potential burden on the health care system.

Undiagnosed COPD and exacerbations. In a population-based study such as CanCOLD we have been able to assess undiagnosed COPD with results showing that two-thirds of subjects with spirometrically-defined COPD had not previously received a COPD diagnosis by a physician ${ }^{20}$. Overall, at least one exacerbation-like event was reported by $40 \%$ of the individuals in the diagnosed group and 22\% in the undiagnosed group $(p=0.001)$. For both subgroups, reporting exacerbation-like respiratory events was associated with lower health status. Factors associated with reporting exacerbation-like respiratory events only in undiagnosed subjects with COPD included decreased lung function, and reporting allergy and anxiety comorbidities; in diagnosed subjects with COPD it included increased dyspnoea, a history of exacerbation-like events at study entry, and reflux-heartburn. However, with respect to the burden of these exacerbations on the health care system, the proportion of hospitalisations, emergency department visits, and unscheduled doctor visits, were similar in individuals with undiagnosed and diagnosed COPD.

\section{Impact of chronic obstructive pulmonary disease: symptom burden and quality of life}

A personalised approach has been proposed by many national and international societies to initiate and escalate/de-escalate a patient's treatment based on their level of symptoms and risk for exacerbations. Symptom burden 
is recommended to be assessed using different questionnaires. Because of its comprehensiveness, the COPD assessment test (CAT) stands as a favourite by many.

The CAT can be used in mild COPD. The CanCOLD study was used to assess how CAT can perform when it is used in mild COPD ${ }^{19}$. The majority of individuals $(72.5 \%)$ were not previously diagnosed with COPD. The mean CAT scores were $5.8 \pm 5.0,9.6 \pm 6.7$ and 16.1 \pm 10.0 for GOLD 1, 2, and 3 and higher, respectively. Higher CAT scores were observed in women, current smokers and ever-smokers, and in subjects with previous diagnosis of COPD. The CAT was also able to distinguish between subjects who experience exacerbations versus no-exacerbations.

Importance of assessing symptom burden in mild COPD. CanCOLD provides a comprehensive overview of work productivity loss (absenteeism and/or presenteeism) among a representative population-based sample of individuals with mild-to-moderate $\mathrm{COPD}^{18}$. Work productivity loss in subjects with a high symptom burden (CAT $\geq 10)$ was about 3-fold higher than in COPD subjects with a low symptom burden $(\mathrm{CAT}<10)$ and in non-COPD subjects. Among the COPD subjects reporting presenteeism, the most frequent type of impairment reported was a difficulty in concentrating (64\%) and a slower than usual pace at work $(64 \%)$, followed by difficulties making decisions $(57 \%)$, postponement of work $(36 \%)$ and the need for assistance (21\%).

Importance of psychological distress in COPD. The CanCOLD study has assessed relationships between levels of psychological distress (depression, anxiety) and poor health behaviours (smoking, reduced physical activity) in individuals with and at risk for COPD ${ }^{21}$. Psychological distress was measured with the Hospital Anxiety and Depression Scale (HADS-A or HADS-D), higher scores defining more disorders. Behaviours included patient self-reports of physical activity level measured by the Community Healthy Activity Model Program for Seniors (CHAMPS) questionnaire, and of pack-years smoking. Results showed elevated depression (HADS-D) in individual with GOLD 3 compared to those with GOLD 1 and 2, and those not categorised as having GOLD score. No effect was seen for HADS-A. Current smoking status was associated with both increased HADS-D and HADS-A. The highest scores were observed in current smokers, and these were significantly higher than scores for never and ever (past) smokers. As well depressive and anxiety symptoms were higher in individuals with lower levels of physical activity.

\section{DISCUSSION}

Clinically relevant new insights from the COLD/CanCOLD study have been expressed with respect to several aspects including the prevalence of COPD, COPD in never smokers, COPD diagnosis using spirometry, COPD misdiagnosis and health care burden, undiagnosed COPD, exacerbations, symptom burden and health status evaluation to personalised treatment in COPD.

A key advantage of CanCOLD is that it uses the same sampling methodology for prevalence assessment as the multinational BOLD study, which was applied across over 25 studies worldwide. This allows for interpretation 
of results not only across Canada but also for their extrapolation to the rest of the industrialised countries. The extent to which results apply to other countries, may be dependent on the degree of similarity in health service organisations and health care access. If we consider that Canada has public and free access to health care for the whole population, the results seen from CanCOLD are not likely to be overestimated compared to other countries with private and/or public-private mixed system. Similarly, implementation of new knowledge into practice can apply differently depending on the country and health care organisation.

The problem of assessing prevalence of COPD by self-reported diagnosis compared with objective spirometrically-defined COPD should be given proper attention in every country. The real prevalence of COPD is much higher than previously estimated by national surveys; there is an underappreciation of the importance of this disease from the public health and from the health care system perspectives.

"Never-smokers" and "ever smokers". Although smoking still remains the major risk factor, "never-smokers" particularly in women account for nearly $30 \%$ of the total burden of COPD in developed countries such as Canada. Thus, physicians should be aware of an increased risk of COPD in "never smokers" reporting a diagnosis of asthma and severe childhood respiratory disease. Our observations also indicate a particular role for passive smoking and exposure to biomass fuel heating as independent factors for COPD, mainly in women. More targeted studies are of course needed to assess the role of other risk factors, including air ambient pollution.
Diagnosis of COPD requires the use of spirometry. It is essential for a family physician to confirm a suspected diagnosis of COPD with spirometry. However, there is considerable variability of spirometry results around the $\mathrm{FEV}_{1} / \mathrm{FVC}$ threshold and a single spirometric assessment may not be reliable for diagnosing COPD, particularly in patients with mild airflow obstruction. The GOLD 2019 Update has recommended that an assessment of the post-bronchodilator $\mathrm{FEV}_{1} / \mathrm{FVC}$ ratio should be confirmed by a repeat spirometry on a separate occasion if the value is between 0.60 and 0.80 , because the ratio may change as a result of biological variation when measured at a later interval. Findings however indicate that if the initial post-bronchodilator $\mathrm{FEV}_{1} /$ FVC ratio is less than 0.60 it is very unlikely to rise above 0.70 spontaneously.

Diagnosis using fixed ratio versus LLN is still a matter of a debate although many national and international societies have recommended using the fixed ratio for symptomatic patients seen in a clinical setting. We have demonstrated in CanCOLD that, from a population perspective, patients meeting either criterion for spirometric confirmation of airflow limitation (fixed ratio or LLN) and in addition having a low $\mathrm{FEV}_{1}$ are the most likely to experience poor outcomes. Physicians should be giving special attention to these patients because they also have increased risk of cardiovascular disease. However, recently from a pool cohort analysis, it has been shown that defining airflow limitation as fixed ratio provide discrimination of COPD-related hospitalisation and mortality that was not significantly different or was more accurate than $\mathrm{LLN}^{23}$. These results support the use of fixed ratio less than 0.70 to 
identify individuals at risk of clinically significant COPD.

The burden on health service uses of undiagnosed and overdiagnosed COPD should be given full attention by physicians in their practice. New knowledge from CanCOLD allowed us to quantify the burden of COPD misdiagnosis, which is quite common, on health services. For patients who are overdiagnosed, in addition to the health services they are receiving for their presumed COPD, their true condition is untreated or undertreated, which is problematic because patients therefore also remain symptomatic. For many, this condition is likely a restrictive lung condition or reactive airways disease, as suggested by other spirometry values. Encounters for cardiac disease may be opportunities to correct the misdiagnosis of COPD. Collectively, these results should serve to advocate for the implementation of strategies to decrease the burden of respiratory disease.

Undiagnosed COPD and exacerbations are underrecognised by physicians and these represent a missed opportunity to diagnose COPD and treat the underlying disease.

Emphasis has been given primarily once COPD has been diagnosed to assess the risk of future exacerbations. Physicians should be giving more attention to any exacerbation-like respiratory events seen in an emergency setting; CanCOLD has shown that these events have substantial impact on health service in individuals with undiagnosed COPD. This is especially important when considering that these are often treated as isolated acute events without awareness of the need for future management of underlying COPD. If these patients were recognised as having COPD, providing proper preventive therapy would have the potential to reduce or prevent complications, such as emergency department visits and hospital admissions.

Symptom burden assessment (using either the modified Medical Research Council questionnaire [mMRC] or CAT) is now recognised as essential in the evaluation of the COPD patient and in the elaboration of a personalised treatment approach. Two measures to assess symptom burden are most widely used, the mMRC that assess dyspnoea and the CAT an 8-item uni-dimensional measure of health status impairment in COPD. Considering that COPD impacts patients beyond just dyspnoea, a comprehensive assessment of symptoms such as CAT is now recommended rather than a simple measure of breathlessness. The CAT can be used in primary care in individuals with mild disease as CAT scores are higher in individuals with worsening airflow limitation and in those with exacerbations. It has been recommended that a symptom score of 10 for the CAT (equivalent to St. George's Respiratory Questionnaire [SGRQ] score $\geq 25$ ) should be used as the threshold for considering regular treatment for symptoms including breathlessness ${ }^{24,25}$. Until recently, substantive evidence for the CAT threshold $\geq 10$ for treatment recommendations was lacking. Using the CanCOLD study we have demonstrated through the assessment of symptom burden of COPD using the CAT scores that a high symptom burden i.e. a CAT $\geq 10$ was associated with experiencing increased losses in work productivity (i.e., combined absenteeism and presenteeism). These patients in clinical practice should be given more attention and treated 
appropriately with maintenance long-acting bronchodilators.

Importance of psychological distress in COPD has been recognised in previous studies. Of note, the CanCOLD longitudinal study is the only one to date to assess the association between symptom levels of psychological distress and health behaviours such as smoking and physical activity among patients with or at risk for COPD. Like other studies, CanCOLD showed that higher symptoms of depression and anxiety were related to higher smoking volume and lower levels of physical activity. Screening for psychological distress and developing interventions that target these health risk behaviours in this population may be important for improving management and outcomes

The results from selected published peer-reviewed articles resulting from CanCOLD longitudinal study or related ancillary and sub-studies have been presented in this review within the context of wide-ranging categorical descriptors based on parameters related to study goals, general or clinical application, emerging research questions or message outcome. The largest contribution of CanCOLD-related publications may be seen to have advanced our understanding of appropriate diagnostic approaches and disease characterisation with impact on recommendations for health care services and interventions. More specifically, the larger proportion of studies to date have served to appropriately quantify COPD prevalence, providing indications for better assessment and diagnosis performance, approaches for early detection, normative data and advice for interventions in primary and secondary care. These findings have also enabled to report on psychosocial correlates of COPD such as quality of life and psychological wellbeing in its various phases of development and its impacts in terms of absenteeism and workforce contributions

From CanCOLD publications we have already learned much, with sufficient substantive data to warrant translation into our clinical practice, guidelines and health policies for the benefit of our COPD patients.

\section{ACKNOWLEDGMENTS}

The authors thank the men and women who participated in the study and individuals in the CanCOLD Collaborative research Group: Executive Committee: Jean Bourbeau, (Mcgill University, Montreal, QC, Canada); Wan C Tan, J Mark FitzGerald; D D Sin. (UBC, Vancouver, BC, Canada); D D Marciniuk (University of Saskatoon, Saskatoon, SASK, Canada) D E O'Donnell (Queen's University, Kingston, ON, Canada); Paul Hernandez (University of Halifax, Halifax, NS, Canada); Kenneth R Chapman (University of Toronto, Toronto, ON, Canada); Robert Cowie (University of Calgary, Calgary, AB, Canada); Shawn Aaron (University of Ottawa, Ottawa, ON, Canada); F Maltais (University of Laval, Quebec City, QC, Canada); International Advisory Board: Jonathon Samet (the Keck School of Medicine of USC, California, USA); Milo Puhan (John Hopkins School of Public Health, Baltimore, USA ); Qutayba Hamid (McGill University, Montreal, Qc, Canada); James C Hogg (UBC James Hogg Research Center, Vancouver, BC, Canada). Operations Center: Jean Bourbeau (PI), Carole Baglole, Carole Jabet, Palmina Mancino, Yvan Fortier, (University of McGill, 
Montreal, QC, Canada); Wan C Tan (co-PI), Don Sin, Sheena Tam, Jeremy Road, Joe Comeau, Adrian Png, Harvey Coxson, Miranda Kirby, Jonathon Leipsic, Cameron Hague (University of British Columbia James Hogg Research Center, Vancouver, BC, Canada). Economic Core: Mohsen Sadatsafavi (University of British Columbia, Vancouver, BC). Public Health core: Teresa To, Andrea Gershon (University of Toronto). Data management and Quality Control: Wan C Tan, Harvey Coxson, (UBC, Vancouver, BC, Canada); Jean Bourbeau, PeiZhi Li, Jean-Francois Duquette, Yvan Fortier, Andrea Benedetti, Denis Jensen (Mcgill University, Montreal, QC,Canada), Denis O'Donnell (Queen's University, Kingston, ON, Canada. Field Centers: Wan C Tan (PI), Christine Lo, Sarah Cheng, Cindy Fung, Nancy Ferguson, Nancy Haynes, Junior Chuang, Licong Li, Selva Bayat, Amanda Wong, Zoe Alavi, Catherine Peng, Bin Zhao, Nathalie Scott-Hsiung, Tasha Nadirshaw (UBC James Hogg Research Center, Vancouver, BC); Jean Bourbeau (PI), Palmina Mancino, David Latreille, Jacinthe Baril, Laura Labonte (McGill University, Montreal, QC, Canada ); Kenneth Chapman (PI), Patricia McClean, Nadeen Audisho, (University of Toronto, Toronto, ON, Canada); Brandie Walker, Robert Cowie (PI), Ann Cowie, Curtis Dumonceaux, Lisette Machado(University of Calgary,Calgary, AB, Canada); Paul Hernandez (PI), Scott Fulton, Kristen Osterling (University of Halifax, Halifax, NS, Canada); Shawn Aaron (PI), Kathy Vandemheen, Gay Pratt, Amanda Bergeron (University of Ottawa, Ottawa, ON, Canada); Denis O'Donnell (PI), Matthew McNeil, Kate Whelan (Queen's University, Kingston, ON, Canada); Francois Maltais (PI), Cynthia Brouillard (University of Laval, Quebec City, QC, Canada); Darcy Marciniuk (PI), Ron Clemens, Janet
Baran (University of Saskatoon, Saskatoon, SK, Canada).

\section{Funding}

The Canadian Cohort Obstructive Lung Disease (CanCOLD) study is currently funded by the Canadian Respiratory Research Network (CRRN); industry partners: Astra Zeneca Canada Ltd; Boehringer Ingelheim Canada Ltd; GlaxoSmithKline Canada Ltd; Novartis. Researchers at RI-MUHC Montreal and Icapture Centre Vancouver lead the project. Previous funding partners are the CIHR (CIHR/ Rx\&D Collaborative Research Program Operating Grants- 93326); the Respiratory Health Network of the FRQS; industry partners: Almirall; Merck Nycomed; Pfizer Canada Ltd; and Theratechnologies. The funders had no role in the study design, data collection and analysis, or preparation of the manuscript.

\section{DISCLOSURES}

Dr. Bourbeau reports grants from CIHR, Canadian Respiratory Research Network (CRRN), Foundation of the MUHC and Aerocrine; personal fees from Canadian Thoracic Society and CHEST; grants and personal fees from AstraZeneca, Boehringer Ingelheim, Grifols, GlaxoSmithKline, Novartis and Trudell; during the conduct of the study.

Dr. Tan reports grants from WCT, Canadian Institute of Heath Research (CIHR/Rx\&D Collaborative Research Program Operating Grants93326), Astra Zeneca Canada Ltd., Boehringer-Ingelheim Canada Ltd, GlaxoSmithKline 


\section{Canada Ltd, Merck, Novartis Pharma Canada Inc., Nycomed Canada Inc. and Pfizer Cana- da Ltd. during the conduct of the study.}

\section{Dr. Perrault has nothing to disclose.}

\section{REFERENCES}

1. Buist AS, McBurnie MA, Vollmer WM et al. International variation in the prevalence of COPD (the BOLD Study): a population-based prevalence study. Lancet. 2007;370:741-50.

2. Bourbeau J, Tan WC, Benedetti A et al. Canadian Cohort Obstructive Lung Disease (CanCOLD): Fulfilling the need for longitudinal observational studies in COPD. COPD. 2014;11:125-32.

3. Tan WC, Bourbeau J, FitzGerald JM et al. Can age and sex explain the variation in COPD rates across large urban cities? A population study in Canada. Int J Tuberc Lung Dis. 2011;15:1691-8.

4. Tan WC, Sin DD, Bourbeau J et al. Characteristics of COPD in never-smokers and ever-smokers in the general population: results from the CanCOLD study. Thorax. 2015;70:822-9.

5. Quanjer PH, Ruppel GL, Langhammer A et al. Bronchodilator Response in FVC Is Larger and More Relevant Than in FEV1 in Severe Airflow Obstruction. Chest. 2017;151:1088-98.

6. Tan WC, Bourbeau J, Hernandez P et al. Canadian prediction equations of spirometric lung function for Caucasian adults 20 to 90 years of age: results from the Canadian Obstructive Lung Disease (COLD) study and the Lung Health Canadian Environment (LHCE) study. Can Respir J. 2011;18:321-6.

7. Tan WC, Bourbeau J, Hernandez P et al. Bronchodilator responsiveness and reported respiratory symptoms in an adult population. PLoS ONE. 2013; 8:e58932.

8. Tan WC, Bourbeau J, O'Donnell D et al. Quality assurance of spirometry in a population-based study -predictors of good outcome in spirometry testing. Copd. 2014;11:143-51.

9. van Dijk W, Tan W, Li P et al. Clinical relevance of fixed ratio vs lower limit of normal of FEV1/FVC in COPD: patient-reported outcomes from the CanCOLD cohort. Ann Fam Med. 2015;13:41-8.
10. Aaron SD, Tan WC, Bourbeau J et al. Diagnostic Instability and Reversals of Chronic Obstructive Pulmonary Disease Diagnosis in Individuals with Mild to Moderate Airflow Obstruction. Am J Respir Crit Care Med. 2017;196:306-14.

11. Diab N, Gershon AS, Sin DD et al. Underdiagnosis and Overdiagnosis of Chronic Obstructive Pulmonary Disease. Am J Respir Crit Care Med. 2018;198:1130-9.

12. Gershon AS, Hwee J, Chapman KR et al. Factors associated with undiagnosed and overdiagnosed COPD. Eur Respir J. 2016;48:561-4.

13. Gershon AS, Thiruchelvam D, Chapman KR et al. Health Services Burden of Undiagnosed and Overdiagnosed COPD. Chest. 2018;153:1336-46.

14. Johnson KM, Tan WC, Bourbeau J et al. The diagnostic performance of patient symptoms in screening for COPD. Respir Res. 2018;19:147.

15. Tan WC, Bourbeau J, Aaron SD et al. Global Initiative for Chronic Obstructive Lung Disease 2017 Classification and Lung Function Decline in Chronic Obstructive Pulmonary Disease. Am J Respir Crit Care Med. 2018;197:670-3.

16. Kirby M, Yin Y, Tschirren J et al. A Novel Method of Estimating Small Airway Disease Using Inspiratory-to-Expiratory Computed Tomography. Respiration. 2017;94:336-45.

17. Tan WC, Hague CJ, Leipsic J et al. Findings on Thoracic Computed Tomography Scans and Respiratory Outcomes in Persons with and without Chronic Obstructive Pulmonary Disease: A Population-Based Cohort Study. PLoS ONE. 2016;11:e0166745.

18. de Sousa Sena R, Ahmed S et al. Work productivity loss in mild to moderate COPD: lessons learned from the CanCOLD study. Eur Respir J. 2017;50.

19. Gupta N, Pinto L, Benedetti A et al. The COPD Assessment Test: Can It Discriminate Across COPD Subpopulations? Chest. 2016;150:1069-79.

20. Labonte LE, Tan WC, Li PZ et al. Undiagnosed Chronic Obstructive Pulmonary Disease Contributes to the Burden of Health Care Use. Data from the CanCOLD Study. Am J Respir Crit Care Med. 2016;194:285-98.

21. Paine NJ, Bacon SL, Bourbeau J et al. Psychological distress is related to poor health behaviours in COPD and non-COPD patients: Evidence from the CanCOLD study. Respir Med. 2019;146:1-9.

22. Pinto LM, Gupta N, Tan W et al. Derivation of normative data for the COPD assessment test (CAT). Respir Res. 2014;15:68.

23. Bhatt SP, Balte PP, Schwartz JE et al. Discriminative Accuracy of FEV1:FVC Thresholds for COPD-Related Hospitalization and Mortality. Jama. 2019; 321:2438-47.

24. Vogelmeier CF, Criner GJ, Martinez FJ et al. Global Strategy for the Diagnosis, Management, and Prevention of Chronic Obstructive Lung Disease 2017 Report: GOLD Executive Summary. Am J Respir Crit Care Med. 2017

25. Vogelmeier CF, Criner GJ, Martinez FJ et al. Global Strategy for the Diagnosis, Management, and Prevention of Chronic Obstructive Lung Disease 2017 Report: GOLD Executive Summary. Eur Respir J. 2017;49. 